\title{
SAND99-0031C
}

\section{FABRICATION AND CHARACTERIZATION OF PZT THIN-FILM ON BULK MICROMACHINED Si MOTION DETECTORS}

\author{
T.J. GARINO, B.A. TUTTLE, G. LAGUNA AND P. CLEM \\ Sandia National Laboratories, MS-1411, Albuquerque, NM 87185-1411
}

\begin{abstract}
Motion detectors consisting of $\mathrm{Pb}\left(\mathrm{Zr}_{\mathrm{x}} \mathrm{Ti}_{(1-\mathrm{x})}\right) \mathrm{O}_{3}(\mathrm{PZT})$ thin films, between platinum electrodes, on micromachined silicon compound clamped-clamped or cantilever beam structures were fabricated using either hot $\mathrm{KOH}$ or High Aspect Ratio Silicon Etching (HARSE) to micromachine the silicon. The beams were designed such that a thicker region served as a test mass that produced stress at the top of the membrane springs that supported it when the object to which the detector was mounted moved. The PZT film devices were placed on these membranes to generate a charge or a voltage in response to the stress through the piezoelectric effect. Issues of integration of the PZT device fabrication process with the two etching processes are discussed. The effects of PZT composition and device geometry on the response of the detectors to motion is reported and discussed.
\end{abstract}

\section{INTRODUCTION}

Integrated, monolithic, high sensitivity piezoelectric devices are needed for a variety of industrial and environmental motion sensor and accelerometer applications. Examples include automobile crash sensors, robotic tactile sensors, industrial vibration measurement devices and thermal flow sensors. ${ }^{1}$ Integration of PZT thin films with micromachined Si structures offers advantages of small size, rugged monolithic packaging, and high sensitivity. Initial calculations $^{2}$ indicate that the PZT thin film // clamped-clamped cantilever beam structures presented in this paper are 1000 times more sensitive per unit volume to acceleration than commercial bulk piezoelectric // mass structures. Another advantage of the use of PZT thin films with Si micromachined, microelectromechanical devices is the generation of much larger forces compared to conventional electrostatic Si micromachined structures. Thus, problems associated with stiction for MEMS devices are substantially reduced or eliminated with the use of PZT thin films.

With this in mind, the design considered for motion detecting applications is shown in Figure 1. This device consists of a clamped-clamped compound beam that is attached to the object whose motion is to be monitored. The thicker region at the center of the beam serves as a

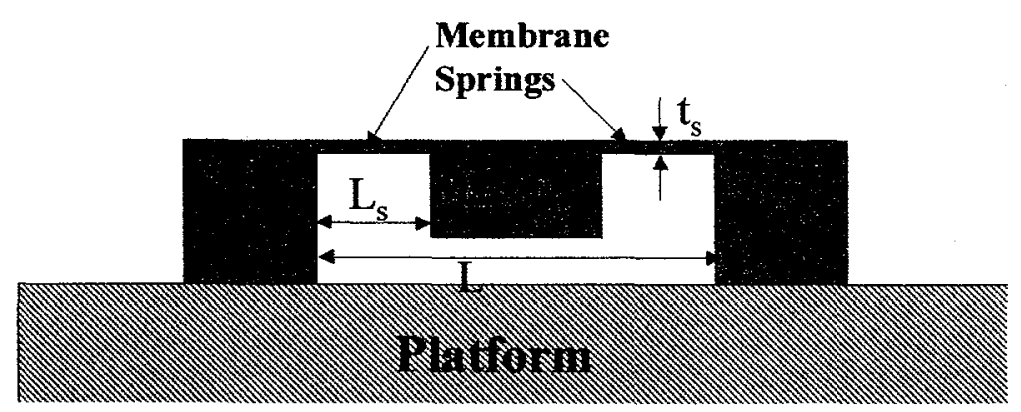

Figure 1. Schematic diagram of a clamped-clamped compound beam used in the design of motion detectors. 


\section{DISCLAIMER}

This report was prepared as an account of work sponsored by an agency of the United States Government. Neither the United States Government nor any agency thereof, nor any of their employees, make any warranty, express or implied, or assumes any legal liability or responsibility for the accuracy, completeness, or usefulness of any information, apparatus, product, or process disclosed, or represents that its use would not infringe privately owned rights. Reference herein to any specific commercial product, process, or service by trade name, trademark, manufacturer, or otherwise does not necessarily constitute or imply its endorsement, recommendation, or favoring by the United States Government or any agency thereof. The views and opinions of authors expressed herein do not necessarily state or reflect those of the United States Government or any agency thereof. 


\section{DISCLAIMER}

Portions of this document may be illegible in electronic image products. Images are produced from the best available original document. 
test mass that is supported by the thinner regions that act like springs. When the object moves, the inertia of the mass creates bending stresses in the thin regions. The stress at the top surface of each of the thin regions varies linearly from maximum tension (compression) at the end near the mass to maximum compression (tension) at the other end, when the object is further from (closer to) the mass than at rest. Through the use of a piezoelectric material, these stresses can be made to generate a charge that can be measured and used to determine the acceleration of the object.

For a simple clamped-clamped beam, one with a uniform thickness, analysis ${ }^{2}$ indicates that the amount of charge generated by the piezoelectric for a given acceleration is proportional to the piezoelectric coefficient of the piezoelectric material, density of the beam material, its width and its length cubed and inversely proportional to its thickness. Analysis of a compound beam indicates that for maximum output, the spring region lengths, $L_{s}$, need to be a quarter of the total length $L$, and the spring thickness, $t_{s}$, needs to be about $1 / 20$ of the thickness of the mass, $t_{m}$. With this geometry, a hundred-fold increase in stress is achieved. When this geometry is coupled with the use of a material with a large piezoelectric response, such as PZT, high sensitivity should be possible with a relatively small device.

Based on these considerations, the motion detector design shown in Figure 2 was developed. In this design, the beam is made from a micromachined silicon wafer. Silicon was chosen because micromachining allows batch fabrication of small structures with precise dimensions and because of our familiarity with depositing PZT sol-gel thin films onto Si wafers. ${ }^{3}$ Two parallel plate structures consisting of bottom electrode, PZT and top electrode are on each thin region since the sign of the stress is opposite in the two halves of each thin region. Two etching techniques were investigated for forming the basic beam shapes: wet etching with hot $\mathrm{KOH}$ and High Aspect Ratio Silicon Etching (HARSE).

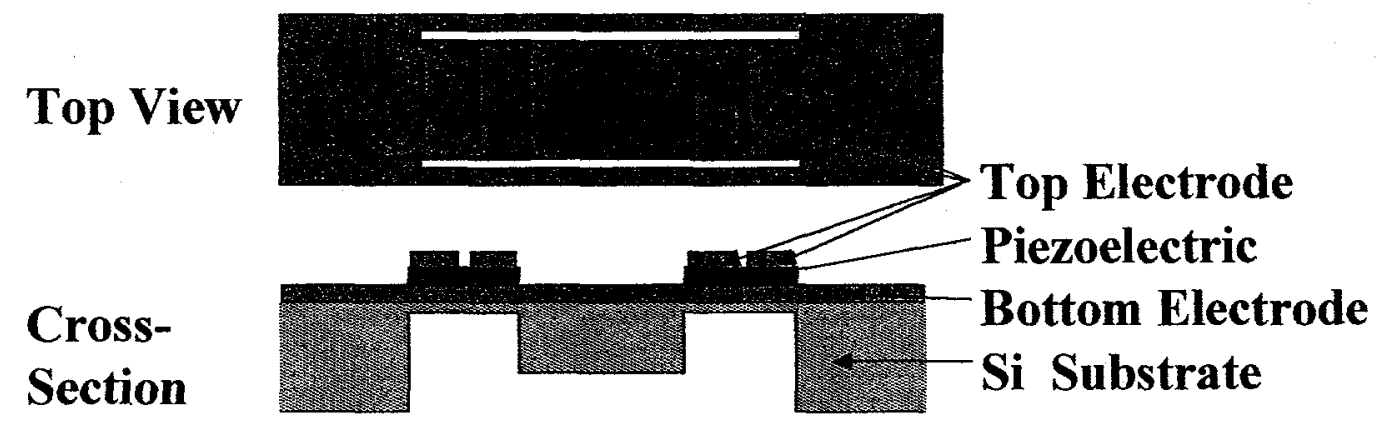

Figure 2. Schematic of a piezoelectric thin-film on micromachined silicon motion detector.

Bulk micromachining of single crystal silicon using hot $\mathrm{KOH}$ solutions has been studied in much detail in recent years. ${ }^{4}$ It relies on the differences in etching rate between the various crystal planes of silicon. The mask material typically used in this process to protect the region of Si not to be etched is silicon nitride made by low-pressure CVD. Also, highly boron doped layers can be used as etch stops since their solubility in $\mathrm{KOH}$ is very low. Using this technique to fabricate motion detectors with PZT films raises several concerns. One is that for standard (100) Si wafers, sidewalls make a $57^{\circ}$ angle with the surface instead of being vertical making the desired geometry unobtainable. Secondly, structures with convex corners, such as the test mass shape desired, are undercut during etching. To get around this problem, corner compensation structures must be used. 5 Finally, the PZT devices on one side of the wafer must be protected while the other side is being etched or the PZT must be deposited onto the fragile etched wafers.

HARSE is a newly developed plasma technique for deep bulk etching of silicon with vertical sidewalls. ${ }^{7}$ In this process, developed by Bosch, standard photoresist is used as a mask 
material since it is etched many times slower than the $\mathrm{Si}$. Unlike the $\mathrm{KOH}$ process, HARSE is non-crystallographic. The etch rate is not effected by the presence of boron and so the etch depth must be controlled by the etch time or by the presence of an etch stop like silicon nitride. For the present application, this process has two concerns. First, the bottom of a deeply etched well is generally somewhat curved instead of being flat as in the case of wet etching. This means that the thin regions of our devices will not be uniform in thickness. Recent work ${ }^{8}$ has made advances to overcome this problem. The second concern is that due to the potential contamination of the etching system with $\mathrm{Pb}$, all etching must be done prior to PZT deposition. This means that PZT and top electrode deposition have to be done on fragile etched structures.

\section{EXPERIMENT}

The first process used to fabricate motion detectors, shown in Figure 3 in a view rotated $90^{\circ}$ from the cross sectional view in Figure 2, was the one in which $\mathrm{KOH}$ etching was used. The starting wafers were obtained from MCNC (Research Triangle Park, NC) and consisted of $10 \mathrm{~cm}$ diameter, $400 \mu \mathrm{m}$ thick (100) Si wafers with $0.5 \mu \mathrm{m}$ of LPCVD silicon nitride on both surfaces and having a highly B-doped region about $5 \mu \mathrm{m}$ from one surface. A $0.3 \mu \mathrm{m}$ thick layer of silicon dioxide was reactively sputtered onto one surface of the wafer to improve the adhesion of the bottom electrode to the wafer. The bottom electrode pattern was defined using a lift-off process. It was desired to have the bottom electrode everywhere except along the sides of the beams where eventually the Si would be completely etched through. An adhesion promoter, HMDS, was spun onto the top of the wafer and then photoresist (PR) was spun on and baked. The wafer was then aligned with the mask such that the beams were parallel to the (110) direction. After exposure and development, the HMDS was removed from the PR-free regions with an ozone-uv etcher. A bottom electrode consisting of $30 \mathrm{~nm}$ of Ti and $170 \mathrm{~nm}$ of Pt was then sputter deposited. The remaining resist was removed with acetone, leaving the electrode only in the desired regions.
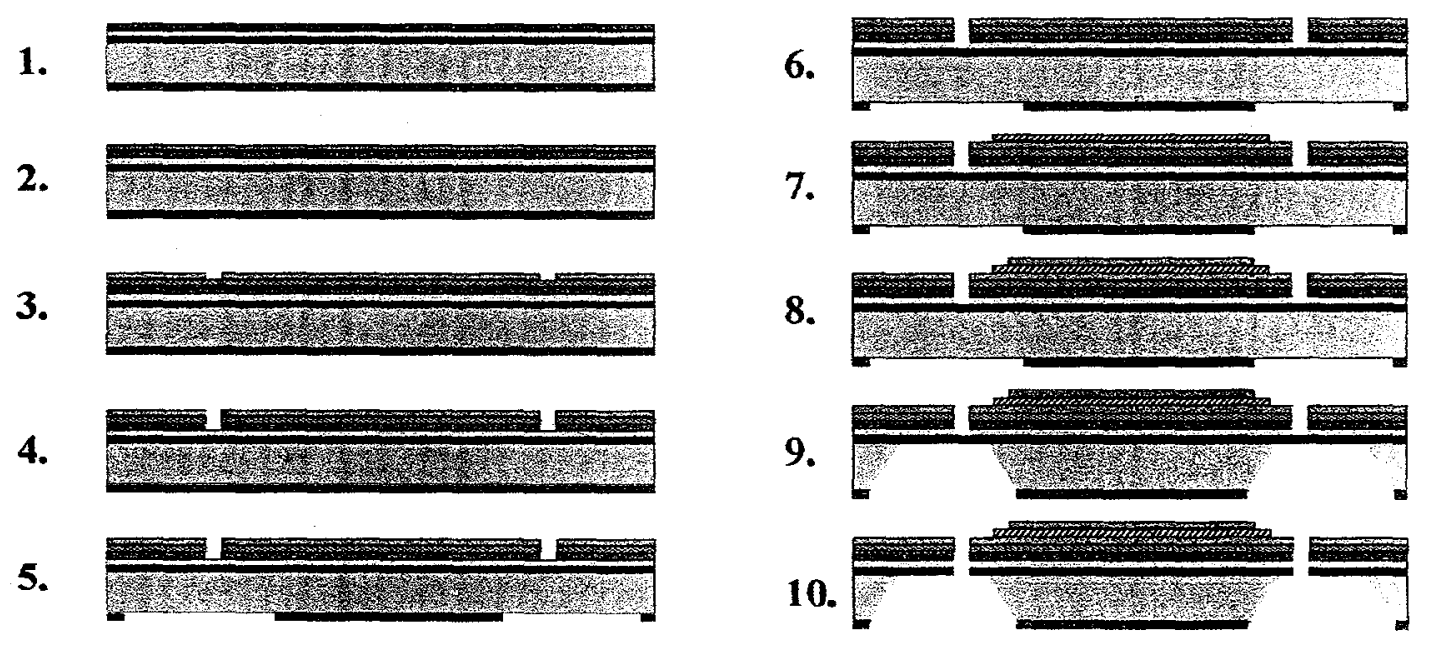

Figure 3. The wet etch motion detector fabrication process: 1) starting wafer with nitride coatings and buried B-doped region, 2) oxide sputter deposited on top, 3) Ti/Pt BEL sputter deposited and patterned by lift-off, 4) nitride and oxide etched in trenches on top by RIE, 5) nitride removed from regions on bottom by $\mathrm{RIE}, 6$ ) $\mathrm{Si}$ in trenches on top etched to $\mathrm{B}$ layer with $\mathrm{HF} / \mathrm{HNO}_{3}$ 7) PZT deposited, 8) top Pt electrode sputter deposited and patterned by lift-off, 9) back etched with hot KOH solution, 10) remaining Si in trenches etched with $\mathrm{HF} / \mathrm{HNO}_{3}$. 
In the next steps, reactive ion etching (RIE) was used to remove the oxide and nitride from the desired regions on the front (the areas that will eventually be etched through) and then to remove the nitride form the desired regions on the back (the areas where the Si will be $\mathrm{KOH}$ etched later). This was done using standard photolithography with appropriate masks to define the desired patterns in PR prior to etching, utilizing front-to-back aligning. The mask used on the back utilized several types of corner compensation features on the convex corners of the test mass areas of the compound beams. In the following step, a $\mathrm{HF} / \mathrm{HNO}_{3}$ mixture was used to etch about $3 \mu \mathrm{m}$ into the Si in the same regions on the front where the oxide and nitride were previously removed. This was done at this point to minimize the amount of etching that will need to be done later to release the sides of the beams by etching completely through the wafer. $P R$ was again used as a mask in the step.

Next, the PZT sol-gel thin film was fabricated by a hybrid metalorganic chemical solution deposition procedure. ${ }^{3}$ The $0.4 \mathrm{M}$ solutions were synthesized by blending $\mathrm{Zr}$ and Ti alkoxide precursors, adding acetic acid and methanol to the solution and then adding the lead (IV) acetate precursor to the blending solution. Additional acetic acid, methanol and distilled water were added to control solution viscosity and improve solution stability. PZT films of $0.8 \mu \mathrm{m}$ thickness were achieved via spin deposition of 8 layers using a crystallization treatment of $650^{\circ} \mathrm{C}$ for $30 \mathrm{~min}$ after deposition of each set of 4 layers. The PZT was patterned using photolithography to define the desired pattern in PR that served as a mask for etching the PZT with an ammonium bifluoride/HCL etchant solution. The top Pt electrode was then sputter deposited and patterned used lift-off.

The next step was to etch the Si from the back side, using the previously defined nitride regions as the mask, down to the $\mathrm{B}$-doped layer using a hot $\mathrm{KOH}$ solution. The front side of the wafer was protected from the $\mathrm{KOH}$ solution by coating it with a protective wax and placing it in a teflon fixture that sealed the front inside the fixture with teflon o-rings. Finally, the $\mathrm{HF} / \mathrm{HNO}_{3}$ mixture was used to etch through the remaining silicon in the trench regions. After etching, the wax was removed with heptane. For this process, the thickness of each test mass region was the same as the original wafer.

The second process used to fabricate the motion detectors, shown in Figure 4, utilized the HARSE process. In this case, standard (100), $10 \mathrm{~cm}$ diameter, $550 \mu \mathrm{m}$ thick Si wafers with 0.3

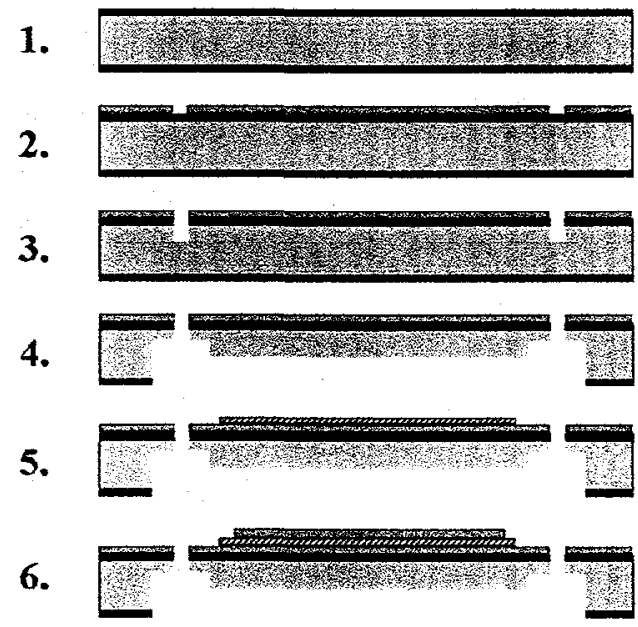

Figure 4. The HARSE motion detector fabrication process: 1) starting Si wafer with thermal oxide coating, 2) Ti/Pt BEL sputter deposited and patterned by lift-off, 3) Si in trenches etched $20 \mu \mathrm{m}$ deep using HARSE after oxide etched with HF, 4) Si etched $285 \mu \mathrm{m}$ deep from back side using HARSE after oxide etched with HF, 5) PZT deposited, 6) top Pt electrode sputter deposited and patterned by lift-off. 
$\mu \mathrm{m}$ of thermal oxide on each side were used. The bottom electrode was deposited as in the first process. Then, PR was patterned so that only the areas without the BEL were exposed and the oxide was removed from these regions with HF. With the same PR layer, the Si in these regions. was next etched down $20 \mu \mathrm{m}$ using HARSE. After appropriate PR patterning of the back side using front-to-back aligning, HARSE was again used to etch the Si down $535 \mu \mathrm{m}$, in two steps, to form the compound beam structure and to intersect the trenches made in the previous step. After the first of the two steps, the PR was developed off of the test mass regions so that these areas were etched along with the membrane areas to the desired thickness. The PZT and TEL were then deposited as in the first process, taking extreme care not to break the fragile wafers during spin coating

Ferroelectric hysteresis properties were measured using a Radiant Technologies Model RT6000HVS tester. Impedance analysis was performed with an HP 4194A impedance analyzer and an HP4284A precision LCR meter. Piezoelectric strain measurements were made with an MTI -2000 Fotonic sensor, which is based on fiber optic array sensing of cantilever beam displacement.

\section{RESULTS and DISCUSSION}

Functional motion detector devices were fabricated using both of the process described. The dielectric constants on the finished devices was around 750. A device made by the HARSE process is shown in Figure 5. The membrane thickness in devices made by this process could be controlled to yield about $15 \mu \mathrm{m}$, although handling them without breaking after the etching was difficult. The $\mathrm{KOH}$ process produced thinner membranes. A signal was generated in the devices in response to an uncalibrated motion.

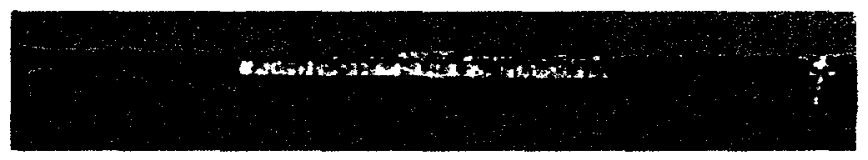

a.

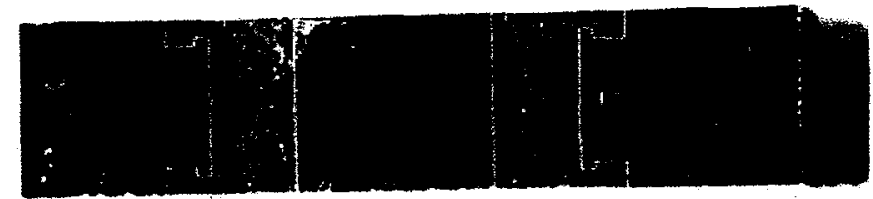

b.

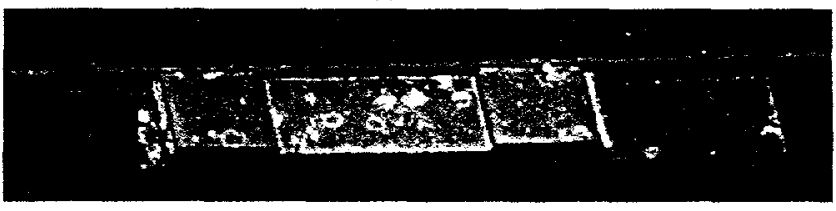

c.

Figure 5. Optical micrographs of motion detectors made by the HARSE process: a) edge view, b) top view and c) back view.

\section{CONCLUSIONS}

Motion detectors consisting of PZT thin films on micromachined Si wafers were fabricated using either KOH etching or HARSE to form the clamped-clamped compound beam geometry. 
Using the HARSE process was simpler and gave straighter sidewalls although thinner membranes were achieved with $\mathrm{KOH}$ etching.

\section{ACKNOWLEDGMENTS}

The authors wish to thank Kent Schubert, Mary-Anne Mitchell, Randy Shul, Susan Von Drasek, Walter Olson, Eric Carleton, Samantha Tuck and Albert Baca for their technical assistance and helpful discussions. This work was performed at Sandia National Laboratories, a multiprogram laboratory operated by Sandia Corp., a Lockheed Martin company, for the US Department of Energy under contract DE-AC04-84AL85000.

\section{REFERENCES}

1. A. Spineanu, P. Benabes, and R. Kielbasa, Sensors and Actuators A 60, 127-33 (1997).

2. G. Laguna, private communication.

3. R.W. Schwartz, B. Bunker, D. Dimos, R. Assink, B. Tuttle, and I. Weinstock, Int. Ferroelectrics, Vol. 2, 243-45 (1992).

4. W. Lang, Materials Science and Engineering, R: Biomimetic Materials, Sensors and Systems R17, p. 1-55 (1996).

5. M. Bao, C. Burrer, J. Esteve, J. Bausells and S. Marco, Sensors and Actuators A 37-38, p. 727-732 (1993).

6. Q. Zhang, L. Liu and Z.Li, Sensors and Actuators A 56, p. 251-4 (1996).

7. R. J. Shul, C.G. Willison, and L. Zhang, SPIE 3511, 252 (1998).

8. A. A. Ayon, C. C. Lin, R. A. Braff, M. A. Schmidt, R. Bayt, and H. H.Sawin, Solid-State Sensor and Actuator Workshop, 41 (1998).

9. P. Luginbuhl, G. Racine, P. Lerch, B. Romanowicz, K. G. Brooks, N. de Rooij, P. Renaud and N. Setter, Sensors and Actuators A 54, p. 530-5 (1996).

10. Y. Nemirovsky, A. Nemirovsky, P. Muralt and N. Setter, Sensors and Actuators A 56, p. 239-49 (1996).

11. C. Lee, T. Itoh, G Sasaki and T. Suga, Materials Chemistry and Physics 44, p. 25-9 (1996).

12. S. Watanabe and T. Fuji, Rev. Sci. Instrum. 67, p. 3898-3903 (1996). 